\title{
Impact of early and standardized treatment with amiodarone on therapeutic success and outcome in pediatric patients with postoperative tachyarrhythmia
}

\author{
Nikolaus A. Haas, $\mathrm{MD}^{\mathrm{a}, \mathrm{b}}$ and Christoph K. Camphausen, $\mathrm{MD}^{\mathrm{a}, \mathrm{c}}$
}

Objective: Effects of timing of inception of amiodarone antiarrhythmic treatment after corrective surgery for congenital heart defects are not yet well defined.

Methods: In a 10-year prospective observational study, 71 of 2651 patients (2885 procedures, 2106 bypass procedures) received amiodarone for newly detected postoperative atrial $(\mathrm{n}=70)$ or ventricular $(\mathrm{n}=7)$ tachyarrhythmia: early treatment (initiation within 60 minutes of arrhythmia detection) and late treatment (after that). From an interim analysis, a treatment protocol was established (32 patients). Inotropic requirements were monitored, as were heart rate, blood pressure, central venous pressure, and sedation requirements.

Results: With early treatment $(\mathrm{n}=29)$, delay before start of amiodarone was $40.7 \pm$ 21.9 minutes, versus $227.7 \pm 228.1$ minutes $(P<.001)$ with late treatment $(\mathrm{n}=42)$. There were significant benefits of early treatment for times to rate $(155.9 \pm 299.8$ vs $407.6 \pm 376.9$ minutes, $(P<.001)$ and rhythm control $(400.4 \pm 845$ vs $1038.5 \pm$ 1158.4 minutes, $P<.001)$, reduction in dose needed for rate control $(28.2 \pm 45.2$ vs $66.5 \pm 137.5 \mathrm{mg}, P<.025)$, and significant reduction in pediatric cardiac intensive care unit stay $(3.32 \pm 1.9$ vs $5.26 \pm 4.27$ days, $P<.01)$. There were continuous improvements in heart rate, blood pressure, and filling pressures without additional inotropic requirements or side effects.

Conclusion: Early treatment of postoperative tachyarrhythmia with amiodarone according to a standardized treatment protocol is safe and has beneficial effects on arrhythmia control and pediatric cardiac intensive care unit stay.

From the Department of Paediatric Cardiac Intensive Care, The Prince Charles Hospital, Brisbane, Australia, ${ }^{a}$ the Department of Congenital Heart Defects, Heart and Diabetes Centre North-Rhine Westphalia, Bad Oeynhausen, Germany, ${ }^{\mathrm{b}}$ and the Department of Paediatric Cardiology, Sydney Childrens Hospital, Sydney, Australia. ${ }^{c}$

Received for publication Nov 10, 2007; revisions received Feb 28, 2008; accepted for publication April 11, 2008.

Address for reprints: Nikolaus A. Haas, MD, Heart and Diabetes Center, North-Rhine Westphalia, Georgstrasse 11, D-32545 Bad Oeynhausen, Germany (E-mail: nikhaas@ hdz-nrw.de).

J Thorac Cardiovasc Surg 2008;136:121522

$0022-5223 / \$ 34.00$

Copyright $(\subset 2008$ by The American Association for Thoracic Surgery

doi:10.1016/j.jtcvs.2008.04.011
A variety of ventricular and supraventricular arrhythmias occur in pediatric patients after corrective surgery for congenital heart defects. Patients admitted to a pediatric coronary intensive care unit (PCICU) have a high incidence of arrhythmias, most likely associated with their underlying pathophysiology and the impact of the surgical intervention performed. ${ }^{1,2}$ In the early postoperative setting, a transient low cardiac output syndrome is common. ${ }^{3}$ Many patients require transient inotropic support with catecholamines, phosphodiesterase inhibitors, or both. Frequently the tachyarrhythmias create hemodynamic instability, necessitating aggressive management to allow beneficial outcome and to reduce ventilation times and stay in the PCICU. ${ }^{4,5}$

Intravenous amiodarone hydrochloride has proved to be an effective antiarrhythmic treatment option for various forms of tachyarrhythmia ${ }^{6-10}$ and is now included in the treatment protocol of the advanced pediatric life support algorithm ${ }^{11}$ for malignant ventricular arrhythmias. Despite the efficacy and good treatment success reported in many case reports and smaller patient series, larger studies on the effects of early treatment in this specific treatment group are missing. We present an analysis of the effects of early and standardized treatment with amiodarone for postoperative tachyarrhythmias on rate and rhythm control as well as on PCICU stay. 


\section{Abbreviations and Acronyms \\ ANZPIC $=$ Australian and New Zealand Paediatric Intensive Care \\ JET = junctional ectopic tachycardia \\ PCICU = pediatric cardiac intensive care unit}

\section{Materials and Methods}

This observational study collected data within the framework of the Australian and New Zealand Paediatric Intensive Care (ANZPIC) data-collection database. The retrospective chart review was approved by the human research committee of the Prince Charles Hospital Health Service District. We electronically searched the computerized database containing all our pediatric intensive care unit admissions for the 10-year period from July 1, 1995, until July 31,2005 , for patients who received amiodarone. In addition to the data required for the purpose of the pediatric intensive care unit quality management (ANZPIC database $\mathrm{e}^{12}$ ), the use of amiodarone was one of the additional selection criteria monitored prospectively in our department. Patients who received amiodarone in the operating room, patients who were already receiving amiodarone treatment before surgery, patients who received amiodarone as part of measures during resuscitation, and patients without surgical intervention were excluded from the analysis.

The diagnosis of arrhythmia was confirmed on the basis of clinical notes, nursing observations in the charts, and independent assessment of the electrocardiograms, including atrial electrocardiograms and electrocardiograms after the administration of adenosine when available, by two experienced pediatric cardiologists and pediatric intensivists (N.A.H. and C.K.C.). The first appearance of the arrhythmia was defined as arrhythmia beginning, and the time between the end of cardiopulmonary bypass and the admission to the PCICU was calculated from that time.

Time of rate control of the tachyarrhythmia was defined as the time at which the spontaneous pulse rate was first recorded at less than $1 \mathrm{SD}$ above the upper end of the reference range for heart rate according to age. If external pacing was used, then the first recorded paced rate less than the value described was taken as the time of rate control. Time of rhythm control of the tachyarrhythmia was defined as the time at which sinus rhythm or successful atrial pacing was documented in the chart.

For the analysis of the impact of the treatment beginning time, treatment groups were divided into early and late treatment groups. Early treatment was the beginning of amiodarone therapy within 60 minutes of first detection of the tachyarrhythmia. Late treatment was the beginning of amiodarone therapy later than 60 minutes after the first detection of the arrhythmia.

Acute adverse events were defined as malignant arrhythmia requiring measures of resuscitation (catecholamine bolus dose or cardiothoracic massage), higher grade atrioventricular block, and most notably the occurrence of significant hypotension, defined as a decrease in blood pressure of more than $20 \%$ of baseline before treatment, necessitating a bolus dose of fluids for volume or bolus administration of catecholamine (epinephrine). As part of the department's nursing protocol, these events should be clearly documented in the monitoring chart.

\section{Treatment}

The management plan for treatment of the tachyarrhythmias was initially at the discretion of the attending intensivist. This included a reduction of catecholamines if possible, correction of biochemical abnormalities, and intravenous infusion of amiodarone. In many cases, a 5-mg/kg bolus dose of amiodarone was administered intravenously during the course of 1 to 4 hours, followed by continuous infusion of amiodarone at a concentration ranging from $5 \mu \mathrm{g} \cdot \mathrm{kg}^{-1}$ $\cdot \min ^{-1}\left(7 \mathrm{mg} \cdot \mathrm{kg}^{-1} \cdot \mathrm{d}^{-1}\right)$ to $15 \mu \mathrm{g} \cdot \mathrm{kg}^{-1} \cdot \min ^{-1}\left(21 \mathrm{mg} \cdot \mathrm{kg}^{-1}\right.$ $\cdot \mathrm{d}^{-1}$ ). Because the Australian consultant system operates without a consultant in pediatric intensive care or pediatric cardiology present in the hospital on a 24-hour, 7-day basis, treatment of tachyarrhythmias was delayed in many circumstances until the definitive diagnosis was established. During the last 3 years of the study, treatment was formalized and changed according to a treatment protocol. This protocol was initially developed for the treatment of junctional ectopic tachycardia (JET) and later modified. ${ }^{13}$

Formal consultant approval before amiodarone therapy was not required once the protocol was in place. The protocol included the administration of amiodarone as a bolus dose of $5 \mathrm{mg} / \mathrm{kg}$ during the course of 1 hour, followed by 1 or 2 further bolus doses of 5 $\mathrm{mg} / \mathrm{kg}$ or by immediate intravenous infusion at $20 \mathrm{mg} \cdot \mathrm{kg}^{-1}$. $\mathrm{d}^{-1}$ until rate control was achieved. For the patient population, rate control was determined as a heart rate below 160 beats/min or $1 \mathrm{SD}$ above the upper end of the reference range for heart rate by age in most cases. A reduction of the heart rate to 140 beats $/ \mathrm{min}$ was sought; the infusion was then tapered to $10 \mathrm{mg} \cdot \mathrm{kg}^{-1} \cdot \mathrm{d}^{-1}$ for at least 24 hours (Figure 1). As part of our standard unit protocol, all patients undergoing bypass surgery received atrial and ventricular pacing wires. As necessary or hemodynamically supportive, atrial pacing was initialized to optimize atrioventricular synchrony.

\section{Parameters}

Patient characteristics were documented, and the age and weight data were used for statistical analysis. The hemodynamic parameters of heart rate, blood pressure, and central venous pressure were recorded continuously in all patients admitted postoperatively. For the purpose of this study, we used the values as documented in the chart

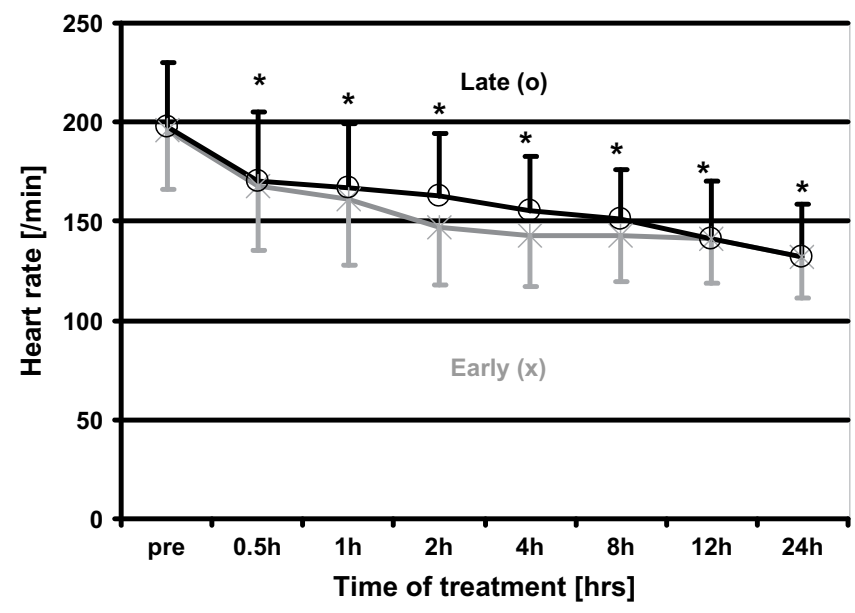

Figure 1. Treatment protocol for postoperative tachyarrhythmia. ECG, Electrocardiography; i.v., intravenously; $i \mathrm{Ca}^{2+}$, ionized calcium current. 
immediately when the arrhythmia became clinically significant (data set before treatment) and at 30 minutes, 1 hour, and 2, 4, 8, 12, and 24 hours after the treatment with amiodarone (data sets at 30 minutes, 1 hour, 2 hours, 4 hours, 8 hours, 12 hours, and 24 hours). The cumulative dose of amiodarone administered was calculated accordingly.

\section{Assessment of Total Inotropic Dose}

The doses of inotropes were analyzed according to the documentation in the chart. For between-group comparisons, a total inotrope dose was calculated by adding the doses of dopamine and dobutamine in micrograms per kilogram per minute and assigning an arbitrary equivalent value of $0.1 \mu \mathrm{g} \cdot \mathrm{kg}^{-1} \cdot \mathrm{min}^{-1}$ inotrope for each 0.1 epinephrine or norepinephrine. ${ }^{14}$ In addition to that, an arbitrary equivalent value of $1 \mu \mathrm{g} \cdot \mathrm{kg}^{-1} \cdot \min ^{-1}$ inotrope was assigned to each $0.1 \mu \mathrm{g} \cdot \mathrm{kg}^{-1}$. min $^{-1}$ milrinone. Data sets used were the same as for hemodynamic parameters (data sets before treatment, 30 minutes, 1 hour, 2 hours, 4 hours, 8 hours, 12 hours, and 24 hours).

\section{Assessment of Total Sedative Dose}

Similar to the calculation of inotropic support, the doses of sedatives and muscle relaxants were analyzed. For between-group comparisons, a total sedative dose was calculated by adding the doses of morphine and midazolam in micrograms per kilogram per hour ( $\mu \mathrm{g}$ equivalent morphine) and assigning an arbitrary equivalent value of $10 \mu \mathrm{g} \cdot \mathrm{kg}^{-1} \cdot \mathrm{h}^{-1}$ sedative for each $1 \mu \mathrm{g} \cdot \mathrm{kg}^{-1} \cdot \mathrm{h}^{-1}$ of fentanyl and an arbitrary equivalent value of $10 \mu \mathrm{g} \cdot \mathrm{kg}^{-1}$. $\mathrm{h}^{-1}$ sedative for each $1 \mathrm{mg} \cdot \mathrm{kg}^{-1} \cdot \mathrm{h}^{-1}$ of propofol. When a paralytic agent was used, an additional arbitrary equivalent value of 10 $\mu \mathrm{g} \cdot \mathrm{kg}^{-1} \cdot \mathrm{h}^{-1}$ sedative was added. Data sets used were the same as for hemodynamic parameters (data sets before treatment, $30 \mathrm{~min}$ utes, 1 hour, 2 hours, 4 hours, 8 hours, 12 hours, and 24 hours).

\section{Statistical Analysis}

Statistical analyses were performed with SPSS version 14 software (SPSS Inc, Chicago, Ill). Continuous variables are expressed as mean $\pm \mathrm{SD}$ (normally distributed data) or as median with interquartile range (nonnormally distributed data). We tested for normal distribution of the data with the Kolmogorov-Smirnov test. Time effects were evaluated with analysis of variance for normally distributed data and the Friedman test for nonnormally distributed data. In case of significant time effects, the paired $t$ test or the Wilcoxon test was used to further specify these differences.

\section{Results}

\section{Patients}

A total of 2651 patients were admitted to the PCICU after cardiac surgery. A total of 2885 procedures were performed, with cardiopulmonary bypass used in 2106 cases. Of those patients, a total of 71 fulfilled the inclusion criteria $(2.46 \%)$.

General. The median age was 3 months, and the median weight was $4.7 \mathrm{~kg}$. Hemodynamically significant sinus tachycardia was present in 3 patients, JET in 37, ectopic atrial tachycardia in 10, intercurrent atrial flutter in 8, atrial fibrillation in 1, intra-atrial reentrant tachycardia in 1, ventricular arrhythmias in 7, supraventricular tachycardia arising from preexisting Wolff-Parkinson-White syndrome in 2, significant atrial ectopic beats or runs in 4 , and combinations thereof in 6 (Table 1). Arrhythmias were seen a median of 270 minutes after admission to the PCICU and a median of 390 minutes after cessation of cardiopulmonary bypass. A $5-\mathrm{mg} / \mathrm{kg}$ bolus dose of amiodarone was administered in 56 of 71 patients, followed by a continuous infusion of about $14 \mathrm{mg} \cdot \mathrm{kg}^{-1} \cdot \mathrm{d}^{-1}$. Rate control was achieved $277 \pm 120$ minutes after the beginning of the amiodarone treatment, and rhythm control was achieved after $685 \pm 300$ minutes (median values; Table 2).

Subgroup analysis. Patient and arrhythmia characteristics for the subgroups of early and late treatment are shown in Tables 1 and 2. There were similar arrhythmias in the subgroups. The groups did not differ significantly with regard to age and weight or as to the beginning of the arrhythmia. There were statistically highly significant positive effects on the times to rate and rhythm control and on the dose required for rate control but not on the total duration and dose of intravenous therapy. Most importantly, there was a significant reduction in the PCICU stay, from 5.26 to 3.32 days.

\section{Hemodynamics}

Heart rate. There was an immediate reduction in heart rate within the first hour after initial treatment; this became

\section{TABLE 1. Patient characteristics}

\begin{tabular}{|c|c|c|c|}
\hline & Total & Early & Late \\
\hline \multicolumn{4}{|l|}{ Cohort selection } \\
\hline Total patients & 2651 & & \\
\hline Procedures & 2885 & & \\
\hline Bypass procedures & 2106 & & \\
\hline $\begin{array}{l}\text { Patients receiving } \\
\text { amiodarone }\end{array}$ & 71 & & \\
\hline \multicolumn{4}{|l|}{ Arrhythmias } \\
\hline Sinus tachycardia & 3 & 1 & 2 \\
\hline $\begin{array}{l}\text { Junctional ectopic } \\
\text { tachycardia }\end{array}$ & 37 & 15 & 22 \\
\hline $\begin{array}{l}\text { Ectopic atrial } \\
\text { tachycardia }\end{array}$ & 10 & 4 & 6 \\
\hline $\begin{array}{l}\text { Intermittent atrial } \\
\text { flutter }\end{array}$ & 8 & 6 & 2 \\
\hline $\begin{array}{l}\text { Intermittent atrial } \\
\text { fibrillation }\end{array}$ & 1 & 1 & 0 \\
\hline $\begin{array}{l}\text { Ventricular } \\
\text { arrhythmias }\end{array}$ & 7 & 2 & 5 \\
\hline WPWSVT & 2 & 0 & 2 \\
\hline $\begin{array}{c}\text { Significant atrial } \\
\text { ectopic beats }\end{array}$ & 4 & 3 & 1 \\
\hline Combinations & 6 & 3 & 3 \\
\hline
\end{tabular}

Time frame was 10 years (July 1995-July 2005. Early, Group with treatment within 60 minutes; late, group with treatment after 60 minutes; WPWSVT, supraventricular tachycardia arising from preexisting Wolff-ParkinsonWhite syndrome. 
TABLE 2. Treatment characteristics

\begin{tabular}{|c|c|c|c|c|}
\hline & Early & Late & $P$ value & Significance \\
\hline No. & $29 / 71$ & $42 / 71$ & & \\
\hline Age (mo) & $13.3 \pm 29.6$ & $18.7 \pm 38.5$ & .637 & NS \\
\hline Weight (kg) & $7.0 \pm 6.0$ & $9.4 \pm 14.5$ & .574 & NS \\
\hline Time after pump (min) & $579.8 \pm 669.2$ & $1099 \pm 1544.3$ & .155 & NS \\
\hline Time after admission (min) & $458.8 \pm 621$ & $927.8 \pm 1480$ & .183 & NS \\
\hline Time to treatment (min) & $40.7 \pm 21.9$ & $227.7 \pm 228.1$ & $<.001$ & $<.001$ \\
\hline Amiodarone bolus dose (No.) & $23 / 29(79.3 \%)$ & $34 / 42(80.1 \%)$ & .875 & NS \\
\hline Pacing (No.) & $12 / 29(41.9 \%)$ & $15 / 42(35.8 \%)$ & .804 & NS \\
\hline Time to rate control (min) & $155.9 \pm 299.8$ & $407.6 \pm 376.9$ & $<.001$ & $<.001$ \\
\hline Time to rhythm control (min) & $400.4 \pm 845$ & $1038.5 \pm 1158.4$ & $<.001$ & $<.001$ \\
\hline Dose at rate control (mg) & $28.2 \pm 45.2$ & $66.5 \pm 137.5$ & .013 & $<.025$ \\
\hline Total time of intravenous therapy (h) & $44.9 \pm 40.3$ & $57.4 \pm 35.8$ & .067 & NS \\
\hline Total intravenous dose (mg) & $247.4 \pm 495.3$ & $253.6 \pm 407.3$ & .808 & NS \\
\hline Pediatric intensive care unit stay (d) & $3.32 \pm 1.9$ & $5.26 \pm 4.27$ & .01 & $<.01$ \\
\hline
\end{tabular}

All values are mean \pm SD except numbers of patients as marked. Early, Group with treatment within 60 minutes; Late, group with treatment after 60 minutes; $N S$, not significant.

statistically significant at 30 minutes (Figure 2). There was, however, no statistically significant difference in heart rate between the subgroups. Pacing was used in 12 of 29 patients $(41.9 \%)$ in the group with early treatment and in 15 of 42 $(35.8 \%)$ in the group with late treatment (difference not significant).

Mean arterial pressure. There was a continuous increase in the mean arterial pressure after the administration of amiodarone (Figure 3) that was statistically significant at 4 hours after the start of treatment. There was, however, no statistically significant difference between the subgroups.

Central venous pressure. There was a continuous but slight decrease in the central venous pressure after the admin-

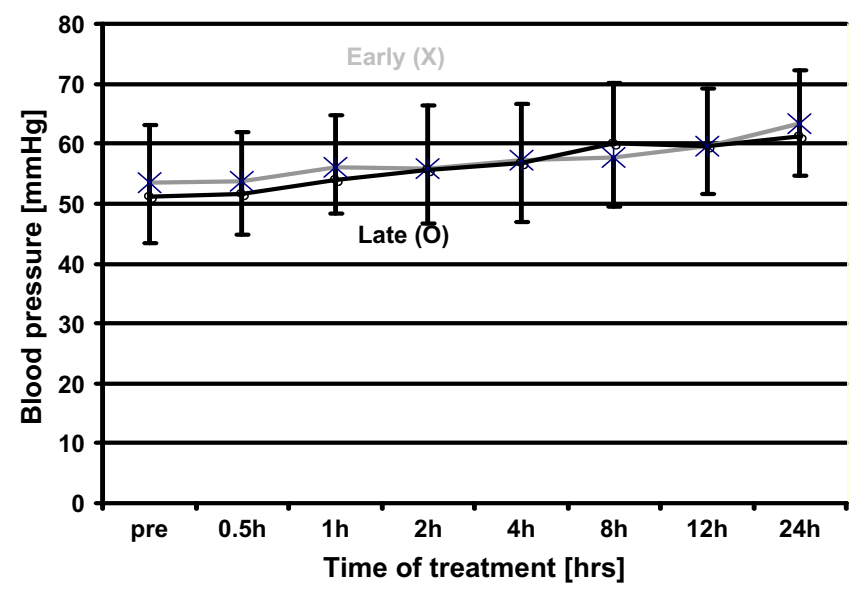

Figure 2. Time courses of heart rate in early treatment group (gray crosses) and late treatment group (black circles). There were significant time effects $(P<.001$ by analysis of variance). No significant differences between study groups were observed. Data points represent mean; error bars indicate SD. istration of amiodarone, from $11.9 \pm 2.9 \mathrm{~mm} \mathrm{Hg}$ before treatment to $10.2 \pm 2.6 \mathrm{~mm} \mathrm{Hg}$ at 12 hours and $9.5 \pm 2.4 \mathrm{~mm} \mathrm{Hg}$ at 24 hours. There was, however, no statistically significant difference between the subgroups.

\section{Drug Requirements}

Amiodarone dose used. The cumulative amiodarone doses used were about $5.1 \pm 6.5 \mathrm{mg} / \mathrm{kg}$ at 120 minutes after the beginning of the treatment and $8.7 \pm 9.6 \mathrm{mg} / \mathrm{kg}$ at 4 hours (Figure 4). There was, however, no statistically significant difference between the subgroups.

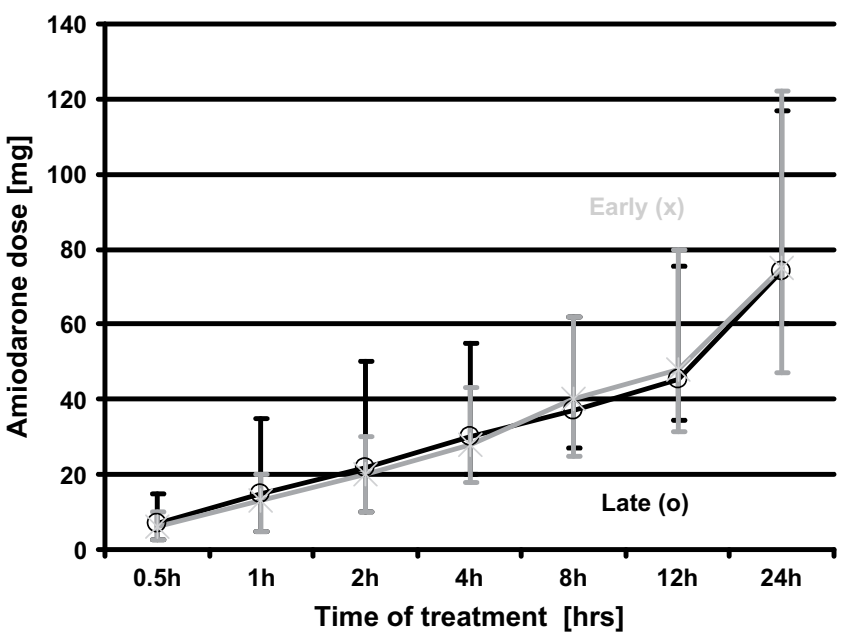

Figure 3. Time courses of mean arterial pressure in early treatment group (gray crosses) and late treatment group (black circles). There were significant time effects $(P<.001$ by analysis of variance). No significant differences between study groups were observed. Data points represent mean; error bars indicate SD. 


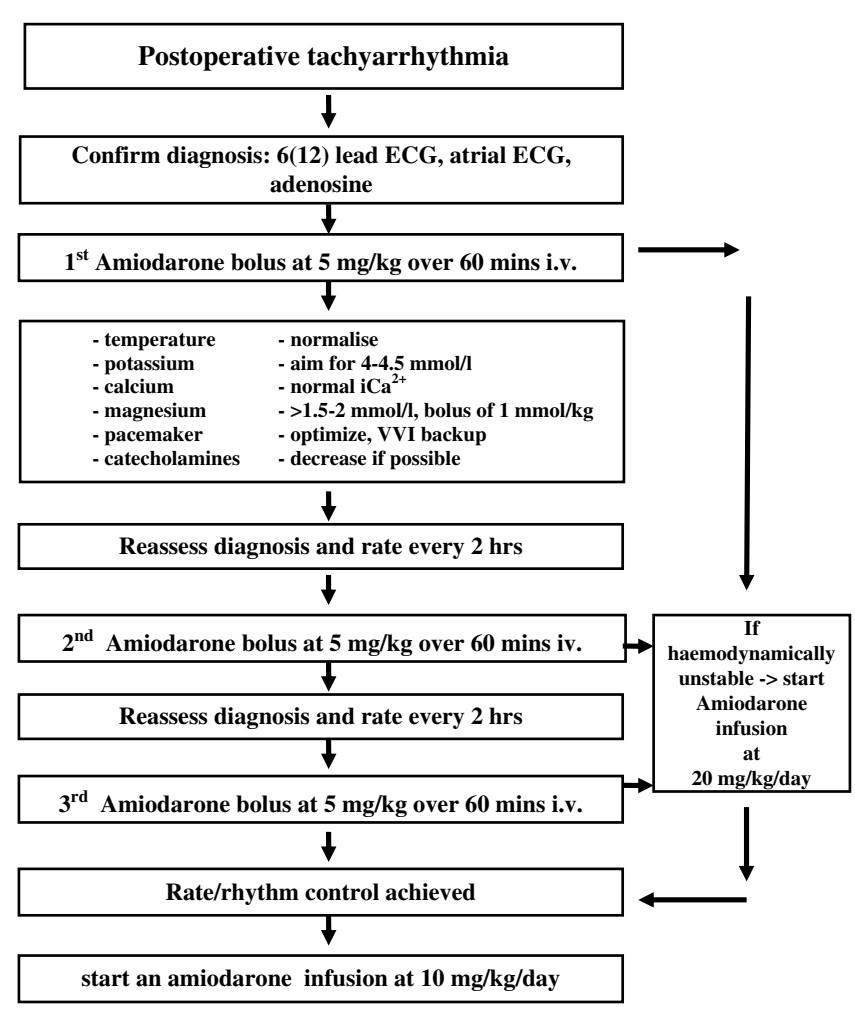

Figure 4. Time courses of cumulative amiodarone dose in early treatment group (gray crosses) and late treatment group (black circles). There were significant time effects $(P<.001$ by Friedman test). No significant differences between study groups were observed. Data points represent mean; error bars indicate SD.

Sedation. With the sedation score used, we could not demonstrate any statistically significant or clinically relevant reduction in the cumulative dose of sedatives used within the initial 12 hours after initial amiodarone treatment. Only at 24 hours after the beginning of amiodarone administration was a significant reduction in sedatives achieved. The mean sedation scores were $26.4 \pm 8.6$ before treatment, $25.3 \pm 6$ at 12 hours after treatment, and $18.7 \pm 5.2$ at 24 hours. There was no statistically significant difference between the subgroups.

Inotropic requirements. With the inotrope score used, at no time could we demonstrate a statistically significant or clinically relevant increase in the cumulative dose of inotropes used. The mean inotropic score was $8.9 \pm 1.7$ ) before initial amiodarone treatment, declining to $7.4 \pm 1.2$ at 12 hours and $5.5 \pm 1.5$ at 24 hours. Again, there was no statistically significant difference between the subgroups.

\section{Adverse Events}

We could not detect in either treatment group any adverse events necessitating additional catecholamine therapy, the administration of additional fluid bolus doses, or resuscitation measures.

\section{Discussion}

This was an observational study of continuously collected data within the framework of the prospective ANZPIC data collection. ${ }^{12}$ We investigated the effects of timing of therapy with intravenous amiodarone in the early postoperative setting in infants and children immediately after corrective or palliative cardiac surgery. To our knowledge, this is the largest study evaluating the beneficial effect of early treatment with amiodarone in this setting. After analysis of the data, there were a number of important findings.

\section{Timing of Amiodarone Treatment}

The administration of amiodarone resulted in a significant and beneficial reduction in the heart rate, facilitating rapid rate and then rhythm control without significant side effects. Adequate rate and rhythm controls are important keystones in postoperative management, because the reduction of heart rate reduces oxygen demands and may facilitate atrial pacing and enable adequate diastolic filling, thereby affecting preload and cardiac output. ${ }^{2,15-17}$ Patients admitted to a PCICU have a high incidence of tachyarrhythmia, most likely associated with the underlying pathophysiology. ${ }^{1,2}$ Tachyarrhythmia, especially atrial fibrillation, is relatively common in adults after coronary artery bypass grafting, leading to significant morbidity including hypotension, heart failure, thromboembolic complications, prolonged hospital stay, and increased hospital costs. Prophylactic administration of amiodarone has been shown to be safe, and effective and hemodynamically well tolerated, even in patients with reduced left ventricular function. ${ }^{18-22}$

In our population, the administration of amiodarone resulted in a significant and beneficial reduction in the heart rate, facilitating rapid rate and then rhythm control. On the basis of our analysis and the definitions we used, we could not detect any significant side effects, such as malignant arrhythmia and, most notably, significant hypotension.

Not all the patients received an amiodarone bolus dose; this variation was initially related to the management plans of the attending consultant intensivists. The overall acceptance of bolus administration increased when the standardized treatment protocol of a "slow" bolus administration of $5 \mathrm{mg} / \mathrm{kg}$ during the course of at least 1 hour was implemented. There was, however, no significant difference in the initial dose of amiodarone administered that could explain the treatment results (Figure 4). In our analysis of the study group that divided the patients into two subgroups (early treatment, within 60 minutes after start of tachyarrhythmia, and late treatment, after 60 minutes), we have demonstrated a beneficial effect of early treatment with regard to rate and rhythm control. There were only small differences in absolute heart rate, but this may be explained by the relatively high percentage of patients receiving atrial pacing as a treatment standard (especially for patients with JET). 
Additionally, we found a reduction in the amiodarone dose needed for rate control, whereas the total dose applied intravenously and the total duration of intravenous treatment were not different. Most importantly, we demonstrated a significant reduction in PCICU stay.

\section{Heart Rate and Rhythm}

We could not discern significant differences between the treatment groups with regard to absolute heart rate at any given point. There was, however, a significant difference in the success rate of treatment as defined. We believe this was an artifact of the relatively high percentage of patients receiving atrial pacing as a treatment standard; this pacing may have altered the actual heart rate achieved, thus explaining the relatively small differences in absolute rate. There was no statistically significant difference in the rate of pacing between the groups (Table 2).

Proarrhythmic reactions to amiodarone and the induction of other acute tachyarrhythmias, especially in patients with prolongation of the QT interval as well as significant bradycardia or atrioventricular block, have been described. ${ }^{23-27}$ In our patient group, no additional arrhythmogenic effects were noticed before hospital discharge. These results are similar to those of other studies in children, which also have not demonstrated any significant arrhythmogenic potential of amiodarone but have reported excellent success rates. Amiodarone can induce sinus bradycardia, sinus arrest, and asystole, especially when used with digoxin, calcium-channel antagonists, or $\beta$-blockers. In the early studies from the Texas Children's Hospital, some patients with atrial flutter had severe bradycardia develop, necessitating pacemaker implantation. $^{28}$

Positive effects were reported by Laird and colleagues, ${ }^{8}$ who treated 11 patients with postoperative JET with a mean bolus dose of $8.2 \mathrm{mg} / \mathrm{kg}$ intravenous amiodarone. Mean heart rate decreased from 202 to 147 beats/min, and mean systolic blood pressure increased from 64 to $88 \mathrm{~mm}$ Hg. Similar effects have been reported in the setting of atrial flutter, repetitive atrial ectopic beats, paroxysms of supraventricular tachycardia, and other forms of supraventricular and ventricular arrhythmia in children. ${ }^{6,7,29-33}$ With the doses and treatment protocol used, we did not see any atrioventricular block, but we did see a significant and beneficial reduction in heart rate.

Three patients after introduction of the treatment protocol had sinus tachycardia as an underlying arrhythmia, 1 in the late treatment group and 2 in the early group. In all 3 cases, other arrhythmias were present simultaneously. We therefore did not demonstrate unnecessary treatment with our protocol.

\section{Hemodynamic Stability}

Tachyarrhythmia often induces hemodynamic instability in patients like ours, necessitating aggressive hemodynamic support. On the basis of the inotropic score necessary to ob- tain adequate hemodynamics, a postoperative low-cardiac output syndrome can be presumed in our patients. Despite this, the inotropic support required could be decreased in both early and late treatment groups, with significant improvement in blood pressure and filling pressures after amiodarone treatment. Amiodarone is known to have significant side effects; these include especially profound hypotension shown in animals and reported in adults, vasodilatation, negative inotropic effects, and significant bradycardia, especially when administered intravenously. ${ }^{6,10,34-36}$ Reports on the administration of amiodarone in the early postoperative setting in children are scarce. In the literature on infants and children to date, many studies have reported only minimal hypotensive side effects. ${ }^{7,8}$ A prospective, randomized, multicenter study of 67 pediatric patients, however, revealed a high rate (mean 36\%) of significant hypotension after rapid bolus administration of amiodarone, and this effect was dose dependent. ${ }^{9}$ Special caution is warranted when treating patients with decreased contractility and end-stage heart failure, and cases of cardiovascular collapse have been reported. ${ }^{37}$ With a slow bolus administration of amiodarone during the course of at least 60 minutes followed by a continuous infusion with 10 to $20 \mathrm{mg} \cdot \mathrm{kg}^{-1} \cdot \mathrm{d}^{-1}$, our protocol proved to be safe in the early postoperative setting without necessitating additional inotropic support. In all cases, treatment initiation with amiodarone was followed about 24 hours later by a reduction in sedation because of hemodynamic stability and in preparation for weaning and extubation.

\section{Limitations of the study}

This study has important limitations. First, it is a singlecenter, uncontrolled, cohort study of pediatric subjects with postoperative arrhythmias treated with amiodarone. Our provocative but statistically significant results may require validation in a larger, and perhaps randomized, prospective trial. Even though the data analysis was performed retrospectively, data acquisition was continuous and prospective in accordance with the prospective and continuous ANZPIC data collection.

One might argue that one of the most important limitations of our study is the lack of effective blinding in this observational investigation, which may have led to a therapeutic bias. Nevertheless, the therapeutic intervention was guided not by the specific amiodarone treatment protocol but by the hemodynamic and other clinical needs of the patients. Other protocols within the unit, especially those that may have had an influence on the results of this study (extubation policies, discharge criteria, catecholamine use, sedation strategies, etc) did not change substantially during the period described. This is especially important with regard to the discharge policies for patients requiring amiodarone treatment and their transfer from the PCICU to a stepdown unit (high-dependency unit or cardiology ward). Other confounding variables that may have contributed to these 
results, such as changes with time in surgical techniques, anesthetic treatment, and bypass management, cannot be excluded.

A number of additional patient-related factors could result in systematic differences in time to treatment, including general hemodynamic status. We did not, however, detect any significant difference in catecholamine requirements, as expressed by the catecholamine score, nor in the baseline blood pressure and other hemodynamic factors. Clearly, there was a trend toward concentration of the patients receiving early treatment in the more recent period (including use of the treatment protocol) and of the patients receiving late treatment in the earlier years. In analysis of these subgroups, however, no clinically relevant differences were evident; that is, early treatment even at the beginning of the study was associated with significantly better results than was late treatment. On the basis of available data, the only major factor that appears to have influenced the outcome in these patients was the early treatment of arrhythmia with amiodarone.

\section{References}

1. Hoffman TM, Wernovsky G, Wieand TS, Cohen MI, Jennings AC, Vetter VL, et al. The incidence of arrhythmias in a pediatric cardiac intensive care unit. Pediatr Cardiol. 2002;23:598-604.

2. Valsangiacomo E, Schmid ER, Schupbach RW, Schmidlin D, Molinari L, Waldvogel K, et al. Early postoperative arrhythmias after cardiac operation in children. Ann Thorac Surg. 2002;74:792-6.

3. Hoffman TM, Wernovsky G, Atz AM, Kulik TJ, Nelson DP, Chang AC, et al. Efficacy and safety of milrinone in preventing low cardiac output syndrome in infants and children after corrective surgery for congenital heart disease. Circulation. 2003;107:996-1002.

4. Hoffman TM, Bush DM, Wernovsky G, Cohen MI, Wieand TS, Gaynor JW, et al. Postoperative junctional ectopic tachycardia in children: incidence, risk factors, and treatment. Ann Thorac Surg. 2002; $74: 1607-11$

5. Dodge-Khatami A, Miller OI, Anderson RH, Gil-Jaurena JM, Goldman AP, de Leval MR. Impact of junctional ectopic tachycardia on postoperative morbidity following repair of congenital heart defects. Eur J Cardiothorac Surg. 2002;21:255-9.

6. Burri S, Hug MI, Bauersfeld U. Efficacy and safety of intravenous amiodarone for incessant tachycardias in infants. Eur J Pediatr. 2003;162: 880-4.

7. Plumpton K, Justo R, Haas N. Amiodarone for post-operative junctional ectopic tachycardia. Cardiol Young. 2005;15:13-8.

8. Laird WP, Snyder CS, Kertesz NJ, Friedman RA, Miller D, Fenrich AL. Use of intravenous amiodarone for postoperative junctional ectopic tachycardia in children. Pediatr Cardiol. 2003;24:133-7.

9. Saul JP, Scott WA, Brown S, Marantz P, Acevedo V, Etheridge SP, et al. Intravenous amiodarone for incessant tachyarrhythmias in children: a randomized, double-blind, antiarrhythmic drug trial. Circulation. 2005;112:3470-7.

10. Perry JC, Fenrich AL, Hulse JE, Triedman JK, Friedman RA, Lamberti JJ. Pediatric use of intravenous amiodarone: efficacy and safety in critically ill patients from a multicenter protocol. $J$ Am Coll Cardiol. 1996;27:1246-50.

11. International Liaison Committee on Resuscitation. 2005 International Consensus on Cardiopulmonary Resuscitation and Emergency Cardiovascular Care Science with Treatment Recommendations. Part 6: Paediatric basic and advanced life support. Resuscitation. 2005;67: 271-91.

12. Slater A, Shann F, ANZICS Paediatric Study Group. The suitability of the Pediatric Index of Mortality (PIM), PIM2, the Pediatric Risk of Mortality (PRISM), and PRISM III for monitoring the quality of pediatric intensive care in Australia and New Zealand. Pediatr Crit Care Med. 2004;5:447-54.

13. Haas NA, Plumpton K, Justo R, Jalali H, Pohlner P. Postoperative junctional ectopic tachycardia (JET). Z Kardiol. 2004;93:371-80.

14. Wernovsky G, Wypij D, Jonas RA, Mayer JE Jr, Hanley FL, Hickey PR, et al. Postoperative course and hemodynamic profile after the arterial switch operation in neonates and infants. A comparison of low-flow cardiopulmonary bypass and circulatory arrest. Circulation. 1995;92: 2226-35.

15. Martinez EA, Epstein AE, Bass EB, American College of Chest Physicians. Pharmacologic control of ventricular rate: American College of Chest Physicians guidelines for the prevention and management of postoperative atrial fibrillation after cardiac surgery. Chest. 2005;128(2 Suppl):56S-60.

16. Martinez EA, Bass EB, Zimetbaum P, American College of Chest Physicians. Pharmacologic control of rhythm: American College of Chest Physicians guidelines for the prevention and management of postoperative atrial fibrillation after cardiac surgery. Chest. 2005;128(2 Suppl): $48 \mathrm{~S}-55$.

17. Walsh EP, Saul JP, Sholler GF, Triedman JK, Jonas RA, Mayer JE, et al. Evaluation of a staged treatment protocol for rapid automatic junctional tachycardia after operation for congenital heart disease. J Am Coll Cardiol. 1997;29:1046-53.

18. Yagdi T, Nalbantgil S, Ayik F, Apaydin A, Islamoglu F, Posacioglu H, et al. Amiodarone reduces the incidence of atrial fibrillation after coronary artery bypass grafting. J Thorac Cardiovasc Surg. 2003;125: $1420-5$.

19. Turk T, Ata Y, Vural H, Ozkan H, Yavuz S, Ozyazicioglu A. Intravenous and oral amiodarone for the prevention of postoperative atrial fibrillation in patients undergoing off-pump coronary artery bypass surgery. Heart Surg Forum. 2007;10:E299-303.

20. Lee SH, Chang CM, Lu MJ, Lee RJ, Cheng JJ, Hung CR, et al. Intravenous amiodarone for prevention of atrial fibrillation after coronary artery bypass grafting. Ann Thorac Surg. 2000;70:157-61.

21. Aasbo JD, Lawrence AT, Krishnan K, Kim M, Trohman RG. Amiodarone prophylaxis reduces major cardiovascular morbidity and length of stay after cardiac surgery: a meta-analysis. Ann Intern Med. 2005;143: 327-36.

22. Kerstein J, Soodan A, Qamar M, Majid M, Lichstein E, Hollander G, et al. Giving IV and oral amiodarone perioperatively fort he prevention of postoperative atrial fibrillation in patients undergoing coronary artery bypass surgery: the GAP study. Chest. 2004;126:716-24.

23. Paul T, Guccione P. New antiarrhythmic drugs in pediatric use: amiodarone. Pediatr Cardiol. 1994;15:132-8.

24. Till JA, Baxendall M, Benetar A. Acceleration of the ventricular response to atrial flutter by amiodarone in an infant with Wolff-Parkinson-White syndrome. Br Heart J. 1993;70:84-7.

25. McGovern B, Garan H, Ruskin JN. Sinus arrest during treatment with amiodarone. Br Med J (Clin Res Ed). 1982;284:160-1.

26. Figa FH, Gow RM, Hamilton RM, Freedom RM. Clinical efficacy and safety of intravenous Amiodarone in infants and children. Am J Cardiol. 1994;74:573-7.

27. Gandy J, Wonko N, Kantoch MJ. Risks of intravenous amiodarone in neonates. Can J Cardiol. 1998;14:855-8.

28. Garson A Jr, Gillette PC, McVey P, Hesslein PS, Porter CJ, Angell LK, et al. Amiodarone treatment of critical arrhythmias in children and young adults. J Am Coll Cardiol. 1984;4:749-55.

29. Raja P, Hawker RE, Chaikitpinyo A, Cooper SG, Lau KC, Nunn GR, et al. Amiodarone management of junctional ectopic tachycardia after cardiac surgery in children. Br Heart J. 1994;72:261-5.

30. Celiker A, Ceviz N, Ozme S. Effectiveness and safety of intravenous amiodarone in drug-resistant tachyarrhythmias of children. Acta Paediatr Jpn. 1998;40:567-72.

31. Etheridge SP, Craig JE, Compton SJ. Amiodarone is safe and highly effective therapy for supraventricular tachycardia in infants. Am Heart $J$. 2001;141:105-10

32. Haas NA, Fox S, Skinner JR. Successful use of an intravenous infusion of flecainide and amiodarone for a refractory combination of postoperative junctional and ectopic tachycardias. Cardiol Young. 2005;15: 427-30. 
33. Perry JC, Knilans TK, Marlow D, Denfield SW, Fenrich AL, Friedman RA. Intravenous amiodarone for life-threatening tachyarrhythmias in children and young adults. J Am Coll Cardiol. 1993;22: 95-8.

34. Cheung AT, Weiss SJ, Savino JS, Levy WJ, Augoustides JG, Harrington A, et al. Acute circulatory actions of intravenous amiodarone loading in cardiac surgical patients. Ann Thorac Surg. 2003;76:535-41.
35. Twidale N, Roberts-Thomson P, McRitchie RJ. Comparative hemodynamic effects of amiodarone, sotalol, and d-sotalol. Am Heart J. 1993;126:122-9.

36. Holt AW. Hemodynamic responses to amiodarone in critically ill patients receiving catecholamine infusions. Crit Care Med. 1989;17: 1270-6.

37. Ng GY, Hampson Evans DC, Murdoch LJ. Cardiovascular collapse after amiodarone administration in neonatal supraventricular tachycardia. Eur J Emerg Med. 2003;10:323-5. 